\section{Mechanisms underlying resilience in ageing}

Yaakov Stern (1), Gaël Chételat, Christian Habeck, Eider M. Arenaza-Urquijo, Prashanthi Vemuri, Ainara Estanga, David Bartrés-Faz, Marc Cantillon, Sean A.P. Clouston, Jeremy A. Elman, Brian T. Gold, Richard Jones, Gerd Kempermann, Yen Ying Lim, Anita van Loenhoud, Pablo Martínez-Lage, Silvia Morbelli, Ozioma Okonkwo, Rik Ossenkoppele, Corinne Pettigrew, Allyson C. Rosen, Nikolaos Scarmeas, Anja Soldan (D), Chinedu Udeh-Momoh, Michael Valenzuela and Eero Vuoksimaa

The recent Opinion article by Cabeza and colleagues (Maintenance, reserve and compensation: the cognitive neuroscience of healthy ageing. Nat. Rev. Neurosci. 19, 701-710 (2018)) $)^{1}$ provides definitions for reserve and maintenance along with a detailed description of compensatory mechanisms from a cognitive ageing perspective. The article represents a much-needed effort to refine the definitions for various forms of compensation. Nevertheless, the definitions presented by Cabeza et al. for reserve, maintenance and compensation diverge from the 'whitepaper' consensus definitions developed by a workgroup of 31 researchers ${ }^{2}$ in ways that we believe are not ideal in terms of their ability to capture the full extent of the mechanisms underlying individual differences in resilience.

Cabeza et al. argue that the differentiation between brain reserve and cognitive reserve should be eliminated, and that those concepts should be replaced by the single, generic term reserve. While it is undeniable that all cognition is brain-based, we disagree with the conclusion that the single term reserve can fully characterize the phenomena that we seek to investigate. All functional imaging observations, including compensation, must consider measures of the underlying brain status. The separation of the terms brain reserve and cognitive reserve therefore serves a critical purpose for empirical research: brain reserve is important for characterizing the physical status of the brain at any point in time (in terms of its volume, cortical thickness, white matter tract integrity and so on), whereas cognitive reserve moderates associations between brain morphology and clinical status (that is, it explains why two people with the same amount of age or disease-related changes differ clinically).

We also advocate retaining the term brain maintenance as opposed to simply using the term maintenance. The specific concept of brain maintenance was proposed based on the observation that genetics and a set of life exposures are associated with reduced development of primary age-related brain changes and pathologies ${ }^{3}$. This clearly separates it from cognitive reserve, which describes resilience against these brain changes.

Finally, we feel that compensation should not be considered at the same level of abstraction as brain maintenance and cognitive reserve. Rather, we believe that compensation is one of many ways that cognitive reserve may be implemented. Thus, the manner and success of compensation is often modulated by lifestyle variables referred to as proxies of cognitive reserve. The authors themselves appear to recognize this issue, stating: "However, complicating the distinction, reserve and the capacity for compensation may interact". In the context of investigating cognitive reserve, investigators might explore whether lifestyle exposures are associated with differential compensation. However, compensation without reference to individual differences and their sources, as well as to brain reserve, yields no insight into how to promote resilience against ageing and disease.

In summary, Cabeza and colleagues perform a valuable service by attempting to establish a common nomenclature for the study of a set of findings noted in functional activation studies, which they group under the overarching term compensation. However, we believe that our recently published paper ${ }^{2}$ presents consensus definitions for brain reserve, cognitive reserve and brain maintenance that will better enable us to pursue the understanding of individual differences and resilience, and generate approaches towards intervention.

There is a reply to this letter by Cabeza, $R$. et al. Nat. Rev. Neurosci. https://doi.org/10.1038/ s41583-019-0139-z (2019).

Yaakov Stern iD ${ }^{1 *}$, Gaël Chételat ${ }^{2}$, Christian Habeck ${ }^{1}$, Eider M. Arenaza-Urquijo ${ }^{3}$, Prashanthi Vemuri ${ }^{3}$, Ainara Estanga ${ }^{3}$, David Bartrés-Faz ${ }^{4}$, Marc Cantillon ${ }^{5}$, Sean A.P. Clouston ${ }^{6}$, Jeremy A. Elman ${ }^{7}$, Brian T. Gold ${ }^{8}$, Richard Jones ${ }^{9}$, Gerd Kempermann ${ }^{10}$, Yen Ying Lim ${ }^{11}$, Anita van Loenhoud ${ }^{12}$, Pablo Martínez-Lage ${ }^{13}$, Silvia Morbelli ${ }^{14}$, Ozioma Okonkwo ${ }^{15}$, Rik Ossenkoppele ${ }^{12}$, Corinne Pettigrew ${ }^{16}$, Allyson C. Rosen ${ }^{17}$, Nikolaos Scarmeas ${ }^{18}$, Anja Soldan iD ${ }^{19}$, Chinedu Udeh-Momoh ${ }^{20}$, Michael Valenzuela ${ }^{21}$ and Eero Vuoksimaa ${ }^{22}$ 'Columbia University, New York, NY, USA. 2Université Normandie, Inserm, Caen, France. ${ }^{3}$ Mayo Clinic Rochester, Rochester, MN, USA. ${ }^{4}$ Universitat de Barcelona, Barcelona, Spain. ${ }^{5}$ RWJ Medical School, New Brunswick, NJ, USA. ${ }^{6}$ Stony Brook University, Stony Brook, NY, USA. University of California, San Diego, CA, USA.

${ }^{8}$ University of Kentucky, Lexington, KY, USA.

${ }^{9}$ Warren Alpert Medical School, Brown University, Providence, RI, USA

${ }^{10}$ German Center for Neurodegenerative Diseases (DZNE) Dresden, Dresden, Germany.

${ }^{11}$ Florey Institute of Neuroscience and Mental Health, Melbourne, Victoria, Australia.

${ }^{2} \mathrm{VU}$ Medical Center, Amsterdam, Netherlands.

${ }^{13}$ Center for Research and Advanced Therapies, CITA-Alzheimer Foundation, San Sebastian, Spain.

${ }^{14}$ IRCCS Policlinico San Martino, Genoa, Italy.

${ }^{15}$ University of Wisconsin-Madison, Madison, WI, USA.

${ }^{16}$ Johns Hopkins School of Medicine, Baltimore, $M D, U S A$.

${ }^{17}$ Stanford University, Palo Alto, CA, USA. ${ }^{18}$ National and Kapodistrian University of Athens, Athens, Greece.

19 Johns Hopkins University, Baltimore, MD, USA.

${ }^{20}$ Imperial College London, London, UK.

${ }^{21}$ Sydney University, Sydney, New South Wales, Australia.

${ }^{22}$ University of Helsinki, Helsinki, Finland. *e-mail:ys11@columbia.edu https://doi.org/10.1038/s41583-019-0138-0

1. Cabeza, R. et al. Maintenance, reserve and compensation: the cognitive neuroscience of healthy ageing. Nat. Rev. Neurosci. 19, 701-710 (2018).

2. Stern, Y. et al. Whitepaper: defining and investigating cognitive reserve, brain reserve, and brain maintenance. Alzheimers Dement. https://doi.org/10.1016/j.jalz. 2018.07.219 (2018)

3. Nyberg, L. et al. Memory aging and brain maintenance. Trends Cogn. Sci. 16, 292-305 (2012).

Competing interests

The authors declare no competing interests. 